\title{
Maximizing microfluidics for in vivo drug screens
}

For Adela Ben-Yakar, going big inherently means going small. Her lab develops microfluidic platforms-tiny 'labon-a-chip' devices-for studying $C$. elegans worms by the thousands. As an NIH Transformative Research Awardee, her group at the University of Texas at Austin had an opportunity to dive deep into developing a new platform for significantly expanding the pace of in vivo drug screening. These efforts, 5 years in the making, have resulted in a large-scale microfluidic screening system that combines high-throughput capabilities with high-resolution imaging (Nat. Commun. 7, 13023; 2016).

"I wanted to develop a drug screening platform for neurodegenerative disease," says Ben-Yakar, " and I quickly came to realize that we need to have a platform which would be very simple to operate, and robust enough so that we could scale it up." Getting there, however, required not only designing a new microfluidic system, but also solving problems across the entire experimental design spectrum. They needed to work out culture protocols for the worms, the most appropriate optics for high-resolution imaging, and an automated image acquisition and processing platform that could analyze large numbers of samples very rapidly.

To start with, wrangling thousands of living worms into precise and pre-determined positions within a couple of minutes for automated imaging is no easy feat. The group redesigned the shape and tapering of the regions where the worms are immobilized (trapping channels), and arranged the channels to maximize the number of worms that could be imaged in a single frame. Using this arrangement, the platform can gather $15 \mathrm{z}$-stack images with micron resolution from an entire $~ 4,000$ set of worms in about 15 minutes.

But speed and resolution were not the only goals; Ben-Yakar hopes that the platform will be easily adopted by others in academia and pharma; even those for whom in vivo drug screening would be a novel method. "I think pharma and researchers are all realizing that using a single assay is not going to get us to clinical success. We need to use multiple tools and models, including small animal models, and we see this device as an enabling technology."

While she hopes the new platform will help push the adoption of in vivo drug screens for neurodegenerative and rare genetic disease using C. elegans, the technology is still pricey. The group is working on new and less expensive ways to build the microfluidic chips, which currently relies on photolithography. "Although our method is certainly much cheaper than trying to do this by hand or any existing method," comments Ben-Yakar, "... you can always make things cheaper so that everyone can benefit from it."

Dustin M. Graham 